\title{
Priming and the retrieval of names from long-term memory'
}

RONALD H. HOPKINS AND RICHARD C. ATKINSON, INSTITUTE FOR MATHEMATICAL STUDIES IN THE SOCIAL SCIENCES, STANFORD UNIVERSITY, Stanford, Calif. 94305

Retrieval from long-term memory was investigated in a task in which $S$ was shown a series of pictures of well-known people and asked to say the surname of the person pictured. For half of the pictures $S$ had learned the correct name in a previous free-recall task, i.e., the name was primed. The results were interpreted to indicate that priming facilitated two aspects of retrieval from memory: search and recovery.

The purpose of this study was to investigate retrieval processes in long-term memory. Each $S$ was given a long series of discrete trials in a picture-naming task. On each trial a picture of a relatively well-known person was presented, and S was to say, as rapidly as possible, the surname of the person pictured. For half of the pictures the appropriate name had been among those studied in a long free-recall list on the previous day; and for half it had not. In addition, for some of the stimuli $S$ was informed at the time of test whether he had or had not studied the correct name during the priming session; and for some of them he was not so informed. It was anticipated that the results could be described by a very simple model of the memory-search process, one incorporating the assumption that priming should facilitate search by limiting the size of the set to be searched.

Suppose that the memory for picture-name pairs was divided into two subsets by the preliminary training: a primed set $A^{\prime}$, and an unprimed set $A$. Further, suppose that $S$ makes exactly $X$ searches in the allotted time, and that he searches $A^{\prime}$ when informed that he had studied the correct name on the previous day, and searches A when informed that he had not. When S is not told whether or not he had studied the correct name, a proportion $t$ of the searches are made in $A^{\prime}$ and the proportion $1-t$ are made in A. Assume that in any single search each item in the searched set is equally likely to be located, and let the function $\mathrm{m}$ indicate the measure of the memory subsets. It is presumed that $m\left(A^{\prime}\right)$ is much smaller than $\mathrm{m}(\mathrm{A})$. Then the predicted probabilities of correct retrieval are: $X /\left[m\left(A^{\prime}\right)\right]$ for the primed-and-informed condition; $X /[\mathrm{m}(\mathrm{A})]$ for the not-primed-and-informed condition; and $[\mathrm{tX}] /\left[\mathrm{m}\left(\mathrm{A}^{\prime}\right)\right]$ and $[(1-\mathrm{t}) \mathrm{X}] /[\mathrm{m}(\mathrm{A})]$ for the primed-and-notinformed and not-primed-and-not-informed conditions, respectively. The present experiment was a test of this simple model.

Method. There were four experimental treatments: informed and primed (Cond. IP); informed and not primed (Cond. IP); not informed and primed (Cond. IP); and not informed and not primed (Cond. $\overline{\mathrm{IP}}$ ). The stimuli were 300 pictures of relatively well-known people. They were selected from books, magazines, and newspapers, and were mounted on $5 \times 8$ file cards. Each $S$ was exposed to half of the stimuli $(150)$, of which 50 were assigned to each of the $I$ conditions and 25 were assigned to each of the $\bar{I}$ conditions. The assignment of stimuli to Ss and treatment conditions was counterbalanced in blocks of 25 stimuli. These blocks were known, on the basis of a preliminary experiment, to be approximately equivalent in overall difficulty.

The priming was done on Day 1 by having $S$ learn the appropriate list of 75 proper names in a free-recall task. The list was presented on a dittoed sheet and $S$ was allowed $8 \mathrm{~min}$ for study, followed by $10 \mathrm{~min}$ to write as many of the surnames as he could remember. This procedure was continued for three alternating study and test periods. Then $\mathrm{S}$ was dismissed, after being told to keep the names well in mind, since he would be doing "something related to the name learning" on the following day.

On Day 2, $\mathrm{S}$ was shown the assigned series of 150 stimulus pictures, one at a time, and was given 5 sec to say the surname of each person pictured. The stimuli were presented in one of three slots on a plywood screen. The slots were labeled, and $S$ was carefully instructed so that he understood that stimuli presented in one of the slots had been primed, that stimuli presented in a second slot had not been primed, and that stimuli presented in the third slot may or may not have been primed. To further minimize the possibility that $\mathrm{S}$ might become confused, stimuli assigned to the various conditions were always presented in the fixed order: IP, $\overline{\mathrm{IP}}$ or $\overline{\mathrm{IP}}, \mathrm{IP}, \mathrm{IP}, \overline{\mathrm{IP}}$ or $\overline{\mathrm{IP}}, \mathrm{IP}$, etc.
Each picture was presented only once. Only one response was permitted and guessing was discouraged. The Ss were 20 university students who were paid for their participation.

Results. The proportions of correctly-recalled names for Trials 1,2 , and 3 of the free-recall priming task were $.44, .64$, and .77 , respectively. Thus, Ss had considerable familiarity with the primed set prior to the picture-naming phase of the experiment.

The proportions of correct naming responses and the mean latencies of these responses were calculated for each $S$ for each condition. The mean proportion correct, mean latency of correct responses, and their standard deviations, are presented in Table 1 for each treatment condition. Principal attention will be directed to proportion correct as the dependent variable. However, it may clearly be seen in Table 1 that the latency of correct responses was shortened by priming. Wilcoxon non-parametric tests indicated this to be a reliable effect in both the $I$ and $\bar{I}$ conditions $(z \geq$ 2.69).

Consider now the results in terms of proportion of correct naming responses, and the search model proposed in the introduction. It may be seen in Table 1 that priming substantially increased the proportion correct in both the I and $\bar{I}$ conditions ${ }^{2}$ ( $\mathrm{z}$ $\geq 3.56$ ). However, the search model did not fare well. Setting $\mathrm{X} /\left[\mathrm{m}\left(\mathrm{A}^{\prime}\right)\right]$ equal to the observed proportion of correct naming responses in Cond. IP, .459, and $[\mathrm{tX}] /\left[\mathrm{m}\left(\mathrm{A}^{\prime}\right)\right]$ equal to the corresponding proportion for Cond. $\overline{\mathrm{I}} \mathrm{P}, .398$, yields an estimate of $\mathrm{t}=.867$. Then, letting $\mathrm{X} /[\mathrm{m}(\mathrm{A})]=.265$, the observed proportion correct for Cond. $\mathrm{I} \overline{\mathrm{P}}$, the predicted proportion in Cond. $\overline{\mathrm{IP}}$ is $(1-.867)(.265)=.035$. This is clearly different from the observed value of .256 . Although more sophisticated estimation procedures could be used, it is obvious that the model is unsatisfactory. Furthermore, a number of other search schemes were tried unsuccessfully. The principal difficulty for any search model seems to be that the proportion correct was greater in Cond. IP than in Cond. $\overline{\mathrm{I}} \mathrm{P}(\mathrm{z}=2.25)$, but that Cond. $\mathrm{I} \overline{\mathrm{P}}$ and $C$ Cond. $\overline{\mathrm{IP}}$ were not significantly different in this respect $(\mathrm{z}=.26)$.

Discussion. In order for a model describing only the search processes to account for these data, it must be quite complex. It has, however, been suggested by many writers that retrieval from memory involves several components. For example, Atkinson \& Shiffrin (1968) have described a search component, a recovery component, and a response generation component. Suppose that the total information in memory, concerning a target item, consists of many bits of information joined by a network of interconnections. In the case of a word or a name, the bits of information might be certain letters, sounds, etc. When an item is "hit" in a search, one of these units of information is located. Then the likelihood that the related bits will be located depends on the strength of the interconnections. The probability of the recovery of enough information for output, once one of the target bits has been "hit," is dependent on the amount of information located and, hence, on the strength of the interconnections. It is reasonable that priming should strengthen these connections, thus facilitating recovery.

Consider, then, a simple two-process model of retrieval in which both search and recovery processes are altered by priming, the search aspects in a manner similar to that described by the original model, and the recovery aspects by a strengthening of the connections between the units of information in memory. Let $\sigma^{\prime}$ be the probability of a hit during search in Cond. IP, and let $\sigma$ be the corresponding probability for Cond. I $\bar{P}$. Also, let

Table 1

Mean Proportions Correct, Mean Latencies of Correct Responses, and their Standard Deviations

\begin{tabular}{lllll} 
Condition & IP & $\overline{\mathbf{I P}}$ & $\overline{\mathrm{I} P}$ & $\overline{\mathrm{IP}}$ \\
\hline Prop. Correct & .459 & .265 & .398 & .256 \\
$\quad$ (SD) & $(.20)$ & $(.18)$ & $(.17)$ & $(.18)$ \\
Lat. of Correct & 2.300 & 2.777 & 2.215 & 2.747 \\
$\quad$ (SD) & $(.49)$ & $(.46)$ & $(.46)$ & $(.70)$ \\
\hline
\end{tabular}


$\sigma^{*}$ be the probability of a successful search in the $\overline{\mathrm{I}}$ conditions. Furthermore, let $\rho^{\prime}$ and $\rho$ be the probabilities of recovery, given successful searches, for the $P$ and $\overline{\mathrm{P}}$ treatments, respectively. Then, the probabilities of retrieval are: $\sigma^{\prime} \rho^{\prime}$ for Cond. IP; $\sigma \rho$ for Cond. I $\overline{\mathrm{P}}$; and $\sigma^{*} \rho^{\prime}$ and $\sigma^{*} \rho$ for Conds. $\overline{\mathrm{IP}}$ and $\overline{\mathrm{IP}}$, respectively. The hypothesis that Cond. I $\overline{\mathrm{P}}$ and Cond. $\overline{\mathrm{IP}}$ were the same in terms of proportion correct could not be rejected. Since these conditions differ only in the search parameter, it may be assumed that $\sigma^{*}=\sigma$. This is not unlikely, inasmuch as it is reasonable that $S$ essentially searches randomly through the entire "picture-name" memory in the $\bar{I}$ conditions; further, the unprimed set is so much larger than the primed set that a search of the former cannot be distinguished from a search of the entire memory.

We are left with the four parameters $\sigma^{\prime}, \sigma, \rho^{\prime}$, and $\rho$. According to his model, the observed difference between Cond. IP and Cond. IP (.459 vs .398) reflects the effects of priming on the search parameter only $\left(\sigma^{\prime} p^{\prime}\right.$ vs $\left.\sigma \rho^{\prime}\right)$; the difference between Cond. $\overline{\mathrm{I}}$ and Cond. $\overline{\mathrm{IP}}(.398$ vs .256) reflects the effect on the recovery parameter only ( $\sigma \rho^{\prime}$ vs $\sigma \rho$ ); and the difference between Cond. IP and Cond. I $\bar{P}(.459$ vs .265) reflects the effects of priming on both the search and recovery parameters $\left(\sigma^{\prime} \rho^{\prime}\right.$ vs $\left.\sigma \rho\right)$. The parameters of the model cannot be separated for estimation from the present data. Nevertheless, it is clear that the model accounts for these findings. Furthermore, this interpretation indicates that, contrary to initial expectations, a large portion of the effect of priming on retrieval may be in the recovery phase.

REFERENCES

ATKINSON, R. C., \& SHIFFRIN, R. M. Some speculations on storage and retrieval processes in long-term memory. Technical Report No. 127, Institute for Mathematical Studies in the Social Sciences, Stanford University, 1968.

\section{NOTES}

1. This research was supported in part by the National Aeronautics and Space Administration, Grant No. NGR-05-020-036.

2. Although guessing from among the known members of the primed set could have augmented performance in Cond. IP, analyses of the overt errors indicated that, with the large primed set employed here, this factor was not an important contributor to correct responding in that condition. 